\title{
Plants Learn and Remember Through Association
}

\section{Mehmet Eraslan*}

Molecular Biology and Genetics, Turkey

*Corresponding Author: Mehmet Eraslan, Molecular Biology and Genetics, Turkey.
Received: November 05,2021

Published: November 25, 2021

(C) All rights are reserved by Mehmet Eraslan.

\section{Abstract}

Plants were researched to discover how they learn and remember without using their brains. The concentrations of specific chemicals ( $\mathrm{K}+\mathrm{Ca}+$, etc. channels), the kind of stress encountered, and the symbiotic connection (mycorrhizal fungal networks) between them all affect learning and memory processes. Based on a study of mycorrhizal fungal networks that connect the roots of trees in forests, there is evidence of tree cognition, microbiome cooperation, and forest intelligence. The plants learnt by reacting to various stimuli in a consistent manner. Many plants can tell the difference between day and night. Plants' biological clocks are sensitive to light, which can cause the rhythm to reset. Plant learning and memory might be aided by epigenetic-related processes. The relevance of epigenetics in these processes stems from its relationship with the environment and the ability to reverse the phenotypic changes more readily it causes as compared to those caused by direct DNA sequence alterations.

Keywords: Ca+ Ions; Brain; Action Potential; Root-brain; Voltage-gated K+ Channels; Molecular Memory; Hopfield-like; Neural Network; Stress; Communication; Mycorrhizal Network; TEs Element; Epigenetic

\section{Introduction}

Learning includes a sequence of biochemical changes in the mammalian brain, including gene transcription regulation. Some of these modifications are stored in post-mitotic cells for the rest of one's life, forming the foundation of long-term memory. Stimuli that cause memory and learning alterations might come from either an endogenous or external source. Several studies have shown that epigenetic processes such as DNA methylation and chromatin modifications are important in learning and memory. Through the modification of chromatin states, the epigenetic machinery provides a mechanism for regulating gene expression in response to specific inputs during learning. Following that, the same mechanism permits the chemical modifications to be maintained during memory operations [1-4].

Plant adaptation to environmental cues and reaction. Because these species appear to be particularly prone to epigenetic changes being passed down across generations, epigenetic variability may play a role in plant adaptation. Environmental signal response and adaptation in plants. Because these species appear to be par- ticularly prone to transgenerational transmission of epigenetic changes, epigenetic variability may play a role in plant adaptation in this setting. This sort of variation is intriguing because it may give plants with a high amount of flexibility, allowing them to learn and remember environmental events $[2,5]$.

Plant memory is divided into three categories based on the length of time it is retained: sensory memory, short-term memory, and long-term memory. The first 0.1 - 3.0 seconds after a stimulus is detected relate to sensory memory. Some sensory memory information can be transmitted to short-term memory in this way. Short-term memory helps you to recall things from a few seconds to a minute without having to think about it. Sensory memory and short-term memory storage capacity and length are typically restricted, meaning that information is available for a limited time but not maintained indefinitely. Long-term memory can retain significantly greater amounts of data for possibly indefinite periods of time, up to the plant's whole life cycle. Storage and recall functions in seedlings, chromatin remodeling in plant development, transgenerational memory of stress, immunological memory of 
tobacco plants and mountain birches, and vernalization and epigenetic memory of winter are just a few examples of memory and learning that have been observed in plants. In addition, Induced resistance and susceptibility to herbivores, memory response in ABA-entrained plants, photo tropically and gravitotropically induced memory in maize, ozone sensitivity of grapevine as a memory effect in a perennial crop plant, mechanical stimulus memory, systemic acquired resistance in plants exposed to a pathogen, and electrical memory are also among the topics covered. Electrical events produce sensory and short-term memory in the Venus flytrap [6-9].

In most situations, the perception of the stimulus is linked to a calcium wave in the cell cytoplasm. The plant's reaction is guided by the properties of the calcium wave (such as its amplitude and duration). More specifically, two types of memory-so-called "learning" (sometimes referred to as "priming" or "training") and "storage/ recall" memory- may allow plants to create a coherent response that is suitable to the whole spectrum of stimuli provided by the environment [10-12].

\section{Electrical activity}

This includes the presence of plant cell cross-walls, plasmodesmata, and neural synapses at root apices; signaling molecules that cross these synapses and transmit information to neighboring cells via calcium-regulated exocytosis and vesicle recycling, like neurotransmitters; and action potentials, which rapidly transmit electrochemical signals to control plant physiology. Trees and plants employ this neuronal physiology to detect the affordances of their surroundings via a variety of sensory organs such as their leaves, roots, and microbiota $[10,12,13]$.

With their "root-brain" hypothesis, Charles and Francis Darwin controversially proposed that the root apex, located between the apical meristem and the elongation zone of a root tip, acts like a brain-like organ that controls plant behavior, like how animals provide support for this hypothesis with the existence of "animal-like sensory-motoric circuits which allow adaptive behavior" such as root crawling $[1,14,15]$.

However, in my opinion, the "root-brain" hypothesis cannot adequately explain the sophisticated plant behaviors observed in roots on its own because, due to their energy efficient cellulose constitution, roots lack the degree of flexibility required to rapidly develop new transient pathways for dealing with unique problems $[14,15]$.
Plants communicate largely by low-molecular-weight chemical molecules, like neurotransmitters in mammals, as well as through the release of $\mathrm{Ca}$ ions from inter- and intracellular storage. Plants exhibit electrical activity as well. When a single leaf on a plant is injured, the experience is communicated with the remainder of the plant within seconds. The activation of glutamate receptorlike genes in Arabidopsis in response to cotton leafworm feeding, Spodoptera lttoralis, causes the production of JA at both local and distant locations in the plant. The receptors are structurally like vertebrate glutamate receptors, which are crucial in the nervous system for fast excitatory synaptic transmission. Electric impulses are largely conveyed through the phloem in plants, like neurological transmission in mammals. As Aart van Bel once pointed out to me, the phloem is electrically isolated from the plant's other tissues. Sieve tubes and their partner cells are found throughout the plant, including the meristems. Because of this conducting tissue, action potentials may travel very instantly to all cells. Although the term "plant neurobiology" was developed to investigate the processing of information that plants acquire from their surroundings, little is known about the importance of electrical impulses in their reactions. Nonetheless, it appears that plants can communicate just as well as mammals. The issue is that they do not comprehend their language (Baluška., et al. 2005, 2009; Gagliano., et al. 2016, 2017; Okano., et al. 2000; Trewavas, 2003, 2009).

Plant Intelligence: What It Is and How It Works According to Trewavas, the coordinating center of a plant is not a brain, but rather a colular network with developing intelligent qualities, and the individual plant is made up of a colular network with emerging intelligent capabilities. Plants are the most complex and clever organisms on the planet at the cellular level. In addition to mitochondria, all green plants include an extra organelle with their own genetic information: chloroplasts, which are derived from blue-green algae. The cell nucleus now controls the creation and assembly of all chloroplast components by correctly regulating and balancing chloroplast protein manufacture from nuclear-encoded and chloroplast-encoded MRNAS. Furthermore, plant cols have structural similarities with neurons, leading to the hypothesis that plant and animal cell-cell communication is comparable $[4,15,19]$.

\section{Electrical activity and memory}

Electrical Memory in the Venus Flytrap: At high temperatures of 28 - $36 \mathrm{C}$, just one mechanical stimulation is necessary to close the trap. The two mechanical stimuli needed to close the trap should 
be provided in a time span of 0.75 to 40 seconds. The cumulative nature of electrical stimulation suggests that the Venus flytrap has short-term electrical memory. It was discovered that ATP hydrolysis generates energy for trap closure, which is then utilized for rapid cation transport. The quantity of ATP in the midrib decreases from $950 \mathrm{M}$ before mechanical stimulation to $650 \mathrm{M}$ after stimulation and closure. However, it is unclear whether electrical stimulation initiates or provides energy to the closure process. The electrical signal from the action potential is sent to the midrib, which might initiate the trap closure. The applied voltage, charge, and electrical current, as well as their amplitude and polarity, were not reported $[10,11,15,20,21]$.

The Venus flytrap has been discovered to have sensory memory. Insects can only contact one or a few trigger hairs for two seconds. This phenomenon is known as molecular or sensory memory in biology. Sustained membrane depolarization was demonstrated to cause a "molecular" memory effect, which has important implications for the biophysical characteristics of voltage-gated ion channels $[10,16,22]$.

Plant Time Sensing and Memory: Circadian Rhythms in Biologically Closed Electrical Circuits Blue light opens the closed inwarddirected $\mathrm{K}+$ channels of extensor cells within 3 minutes during the dark phase. Blue light, on the other hand, closes the inward-directed $\mathrm{K}+$ channels of flexor cells, which are open in the dark. However, during the light time, the issue becomes more complicated. Memristors are memory circuit components whose characteristics are determined by the system's history and state. Between Phyto sensors and Phyto actuators, memristors can help with electrical signal transmission across plasma membranes. A pharmacological study claims that one of these memristors might be a voltage gated $\mathrm{K}+$ channel. Electrical memory in plants can be aided by memristors. Electrical components are seen in the Venus flytrap's sensory and short-term memory $[4,11,15,23,24]$.

The presence of memristors in neural networks, voltage-gated channels, synapses, and the brain has been demonstrated theoretically. Plants may contain memristors because they have voltage-gated $\mathrm{K}+$ channels that are comparable to those seen in mammals. Memristors have recently been discovered as components of plasma membranes in a variety of plants, flowers, fruits, and seeds. In plant tissue, tetraethylammonium chloride, a voltage-gated $\mathrm{K}+$ channel inhibitor, or 5-nitro-2-(3-phenylpropylamino) benzoic acid (NPPB), a voltage-gated $\mathrm{Cl}$ - and $\mathrm{K}+$ channel blocker, converts a memristor to a resistor. The finding of memristors in plant membrane structures opens new avenues for modeling and understanding electrical processes in these structures. When memory elements are pinched hysteresis loops are subjected to a periodic stimulus, they can either cross (memristor type 1 ) or not (memristor type 2) [11,13,17,25].

In the squid giant axon, Hodgkin, and Huxley (1952) developed a membrane model that accounts for $\mathrm{K}+, \mathrm{Na}+$, and ion leakage channels. Each ion species' membrane resting potential is considered as a battery, and the degree to which the channel is open is represented by a variable resistor. The Hodgkin-Huxley time-varying potassium conductance, according to Chua, is a first order memristor, while the Hodgkin-Huxley time-varying sodium conductance is a second order memristor. In a single voltage-gated sodium channel, Nayak and Sikdar (2007) discovered time-dependent molecular memory. Volkov and Markin (2016) and Volkov., et al. (2017) discovered memristors in voltage-gated $\mathrm{K}+$ channels present in plants, seeds, fruits, and flowers [11,13,15,24,25].

\section{Accommodation to their environment}

A plant germinates and establishes itself in a certain spot, which becomes its permanent position and where it must respond to signals created by the dynamics of various environmental circumstances. In most situations, the perception of the stimulus is coupled with a calcium wave in the cell cytoplasm. The properties of the calcium wave (such as its amplitude and length) are considered to assist the plant in responding appropriately. More specifically, two types of memory-so-called "learning" (also known as "priming" or "training") and "storage/recall" memory-may enable plants to create a coherent response that is suitable to the whole spectrum of stimuli provided by the environment [26-29].

The "Learning" Form of Plant Memory: In the learning form, repetition of the same stimulus progressively modifies the strength of the reaction, either negatively or favorably, and such behaviors may be labeled "familiarization" (alias "habituation") or "sensitization", respectively, by comparison with animal physiology. As an example of familiarization, after being treated to a number of gusts of wind, tobacco plantlets transiently cease to produce a cytosolic calcium wave following another gust of wind. Another example is shown in Arabidopsis seedlings, where the cytosolic calcium wave produced by cold shock is reduced when it is preceded by pro- 
longed or repeated cold treatments. Sensitization occurs when the cytosolic calcium wave produced following a hyperosmotic stress (mimicking drought stress) is enhanced in plants that have previously been exposed to hyperosmotic stress. The evolutionary advantage of learning memory is that plants can economize responses to nonthreatening stimuli (in the event of familiarization) while giving greater responses to harmful stimuli and stressors (in the case of sensitization). It was also shown that oxidative stress pretreatment reduces the increase in cytosolic calcium concentration caused by hyperosmotic stress in Arabidopsis seedlings, suggesting that the combination of multiple types of stress might create new responses. (Plant illustrations) Plant Memory in the "Storage/ Recall" Mode: This might be due to a simple locking/unlocking of genes $[9,15,18,21,23,25,29]$.

Indeed, numerous different groups have discovered that epigenetic regulation via DNA methylation/demethylation processes is related with the perception of stimuli and stressors. In terms of the store/recall type of memory, long-term information storage has been demonstrated in bryony (Bryonia dioica), a common cucurbitaceous plant that climbs in bushes and hedges. The woody plant is another example of long-term storage (Rosa hybrida) $[2,7,8,23]$.

\section{Mycorrhizal network}

Mycorrhizal fungal networks that connect the roots of trees in forests are becoming more recognized for their ability to allow inter-tree communication via resource, defense, and kin recognition signals, and therefore impact the complex behavior of neighbors. These tree activities contain cognitive characteristics, such as perception, learning, and memory, and they impact plant features that are indicative of fitness. They provide evidence that mycorrhizal network architecture is similar to brain network topology, with scale-free patterns and small-world characteristics associated with local and global efficiency, both of which are important in intelligence [15].

Furthermore, the diverse exploration techniques of interconnected fungal species are analogous to crystallized and fluid intelligence, both of which are crucial in memory-based learning. The biochemical signals that travel between trees via fungal connections are considered to give resource subsidies to receivers, particularly among regenerating seedlings, and some of these signals appear to be neurotransmitter-like $[1,15]$.
The present instances of adjacent tree behavioral, learning, and memory responses are enhanced by mycorrhizal network communication, including, respectively, (1) improved seedling survival, development, nutrition, and mycorrhization in the understory, (2) heightened defense chemicals and kin selection, and (3) collective memory-based interactions between trees, fungus, salmon, bears, and humans that improve the overall health of the forest ecosystem. Viewing this data through the perspective of tree cognition, microbiome partnerships, and forest intelligence may lead to a more holistic approach to understanding ecosystems, as well as more human empathy and concern for the health of their forests. To fulfill plant nutrition and water demands, roots and fungal hyphae must explore wide areas of soil to collect limited and patchy supplies, requiring cognitive activities such as decision-making, search and escape movements, and neighbor identification $[14,15,28]$.

Trees have been shown to sense and communicate with one another and with other plants via root channels or aerial messages. Within- and between-plant communication, according to Baluka and Mancuso, is primarily accomplished via signal transport within and between roots, where compounds such as auxins serve as neurotransmitters across synapses at cell cross-walls within roots, across synapses between the apices of different plant roots, or between plant roots and symbiotic microbes and fungi in the rhizosphere. Because all trees are mycorrhizal in nature, and mycorrhizal networks are thought to be widespread in forests, this suggests that mycorrhizas mediate the majority of belowground communication between trees in nature and that mycorrhizal networks are intimately engaged in tree cognition $[4,11,17,30]$.

This closely follows the discovery of Baluka and Mancuso that plant communication, as well as the involvement of cell-to-cell synapses and neurotransmitter-like chemicals, coevolved with microbes. However, much of the previous research on plant communication and cognition has been performed on non-mycorrhizal plants produced in the lab or has not reported on the involvement of mycorrhizal fungus. The purpose of this chapter is to examine the fundamental role of mycorrhizal fungal networks in tree communication, as well as the functional, ecological, and evolutionary relevance of this communication to forest ecosystems in nature. It will present examples from the lab's research to discuss existing experimental evidence for cognition among trees assisted by mycorrhizas. The aim is that this will lead to a more integrated 
approach to researching plant cognition in natural environments, including plant microbiomes [4,12,14-16].

Behavioral responses, learning, and memory in plants

Plant behavior responses and learning are actions or changes in plant morphology and physiology in response to external stimulithese are caused by the cognitive agents, which include their sensors, mycorrhizal networks, and signal transmission, as previously stated. These agents provide sophisticated mechanisms for plants to perceive their environment, storing information in memory banks such as annual growth rings, seeds, or branching, rooting, and network topologies, and using this information for memorybased learning, which drives behaviors such as choice, decisionmaking, defense, and neighbor recognition $[8,15]$.

Plant communication via mycorrhizal networks, for example, has been linked to behavioral changes such as changes in rooting patterns, mycorrhizal network growth, nutrient absorption, and defense enzyme synthesis. These modifications have affected the transmitter and recipient plants' survival, development, and fitness. McNickle., et al. (2009) describe behavior as a decision point manifestation of plant plasticity, with each option involving tradeoffs that impact fitness $[3,15,20]$.

\section{Learning}

Semchenko., et al. (2007), demonstrated that root exudates conveyed specific information about neighbors' genetic relatedness, population origin, and species identification, and that locally applied exudates elicited distinct root behavior responses in neighbors. This included greater root density, which was achieved by morphological modifications rather than biomass allocation, implying that the plants learnt from their neighbors to reduce the energy cost of their activity. Because mycorrhizal fungus and mycorrhizal networks are ubiquitous in nature, any root exudates implicated in kin recognition are likely to be filtered by mycorrhizal fungi and mycorrhizal networks. Recent research employing stable-isotope probing discovered that mycorrhizal networks transferred more carbon from older donor Douglas-fir seedlings to the roots of younger kin receiver seedlings than to stranger receiver seedlings, implying that genetically related neighbors had a fitness benefit $[2,5,15,25]$.

Defense signals traveling through mycorrhizal networks cause recipient plants to respond quickly, as evidenced by abrupt chang- es in foliar defense chemicals and insect resistance. Broad beans (Vicia faba), for example, responded to aphid assault by rapidly transmitting defense signals via mycorrhizal networks to neighboring bean plants, which learnt from this and produced aphid-repellent compounds and aphid-predator attractants. Defoliation of Douglas-fir resulted in the simultaneous transmission of defensive signals and carbon to nearby healthy ponderosa pine via mycorrhizal networks, according to another research. These signals taught the ponderosa pine to enhance defense enzyme synthesis and protect itself from the loss of healthy hosts $[4,15]$.

As previously demonstrated, increases in mycorrhizal networkmediated enzyme synthesis result from activation of defense genes and modification of gene expression, resulting in an epigenetic impact. EMF, for example, has decreased significantly in areas of western North America severely damaged by mountain pine beetle-induced dieback of lodgepole pine (Pinus contorta). Seedlings cultivated in soil from beetle-infested pine stands learnt from this decrease and then displayed lower biomass and monoterpene production compared to those grown in soil from undisturbed pine stands. This demonstrates a fungal symbiont-mediated transgenerational cascade including learning, memory, and Epigenetics $[3,5,7,15]$.

\section{Memory}

The recent research in the Pacific Coast salmon forests has yielded an intriguing example of memory-based learning. Researchers are currently investigating how "mother trees"-the old cedars, spruces, and firs of the Pacific Coast-transmit nutrients across the forest via vast fungal networks, nourishing the entire ecosystem. These animals bring their prey up the riverbanks, where they feast on the safe, warm, and dry benches beneath the mother trees of the riparian forest. As a result, the nutrients in the salmon corpses, as well as their excrement and urine, are distributed. In safety, the bears consume the intestines, allowing the carcasses to decompose and the nutrients to soak into the soil. Mycorrhizal fungi linked with tree and plant roots absorb essential nutrients from the soil and utilize them to supply 25 - 90\% of the tree and plant nitrogen needs. Once the salmon nutrients have been digested in the woody tissues of the trees, they are retained in tree rings for millennia, giving a memory bank of past salmon runs for as long as the tree is old. This mechanism contributes to quicker tree development along salmon streams and is responsible for the large size and exceptional productivity of these ancient forests. It has also been 
demonstrated to influence the variety and composition of plant, insect, and bird populations. This process of salmon nutrient pickup by mycorrhizas, storage in tree rings, and retrieval of the information for tree development is a forest memory $[5,15,16,25]$.

The transmission of the salmon memory, the telling of the narrative through their communication networks, enables the trees, fungus, bears, and fish to collaborately inform the ecosystem's production and health. These lush woods, in turn, shade and nourish the salmon rivers, regulating water temperature and transporting nutrients to the ebb tides via seepage, producing a positive feedback loop that supports fish health and productivity. The Aboriginal people gather the tree parts-the bark and roots, which are created with salmon nitrogen-to manufacture clothes, art, and tools, such as those used for salmon harvesting. Mother trees are essential in completing this loop $[5,15,25]$.

\section{A basic hopfield-like cellular network model}

Parallel and distributed processing are also used by neural networks, which are mathematical representations of the brain. The behavior of the dodder coil is connected to the emergence of intelligence as a macroscopic function, but it must examine its reasoning on a microscopic level. Almost 80 years ago, J.C. Bose (1923) discovered electrical communication between plant cells. Since then, there have been many reports of crosstalk across biochemical signaling pathways in plants. Chakrabarti and Dutta exploited the threshold behavior of plant cell membranes to describe gates for executing basic logical operations. In contrast to the randomly dispersed positive-negative synaptic connections in real brains, they observed that plant network connections are either all positive (excitatory through neuronal states) or all negative (inhibitory). This chapter presents several research and observations regarding the current-voltage characteristics of the plant cell membrane. To represent a plant with such characteristics, researchers utilized a Hopfield-like network model containing both ferromagnetic retrieval and anti-ferromagnetic components. They then utilized the replica approach to evaluate the model [1,20,31,32].

\section{The I-V properties of cell membranes}

Many observations on the properties of plant units (cells) are mentioned in this section, especially the current (I)-voltage characteristics of their cell membrane. They demonstrated the normal non-linear I-V characteristics of cell membranes acting as logical gates in a single cell. They thought about how the kind of cell membrane impacts its ability to transmit electrical impulses [20,31].
The output of the i-th unit Oi in the Hopfield model is supplied as a +- randomly distributed weight matrix in terms of the Hebbian rule, but in input-output logical units such as perceptron's for neural networks, it is presented as where the strength of each connection wij is all positive or negative. Based on these experimental discoveries and fundamental observations, researchers now ask a logical question: might plants act as memory devices similar to human brains? [20,31].

As a non-linear unit, Chakrabarti and Dutta (2003) created a mathematical plant cell. The I-V properties of cell membranes acting as logical gates were discovered to be equivalent to those of the Zenner diode. Could the plants function as memory devices in the same way that a human brain does? Obviously, there is no frustration in the foregoing concept of a single unit in the plant, as there is in animal brains. This section aims to explain how limitations on the sign of the weight matrix affect plants' capacity to recover patterns as associative memory $[1,20,31]$.

\section{Epigenetic}

Several studies indicate that epigenetic-related processes may play an important role in plant learning and memory. The significance of epigenetics in these processes stems primarily from its relationship with the environment and the ability to reverse the ensuing phenotypic alterations more readily as compared to those connected with direct DNA sequence changes $[2,7]$.

Learning is accompanied by a variety of molecular changes in the mammalian brain, including gene transcription regulation. Some of these modifications are kept in post-mitotic cells for the rest of one's life and serve as the foundation of long-term memory. Endogenous or external stimuli can cause alterations in memory and learning. Several studies have found that epigenetic processes such as DNA methylation and chromatin modifications play an important role in learning and memory. The epigenetic apparatus, which modulates chromatin states, provides a mechanism for regulating gene expression in response to specific inputs during learning. As a result, the same mechanism allows for the preservation of molecular modifications throughout memory operations $[1,5,7,33]$.

Plant reaction and adaptation to environmental cues. In this respect, epigenetic variability may be important in plant adaptation since these organisms appear to be particularly susceptible to epigenetic changes being passed down across generations. Plant 
reaction and adaptation to environmental cues. In this respect, epigenetic variability may be important in plant adaptation since these organisms appear to be particularly susceptible to epigenetic changes being passed down across generations. This sort of variation is quite interesting because it may offer plants with an extraordinary amount of flexibility, functioning as a mechanism that allows plants to learn and remember environmental events. The epigenetic process is linked to three major factors: cytosine methylation, TEs, and Histon modification $[2,3,7]$.

However, in this situation, as with genetic variation, the process leading to transgenerational transfer of phenotypes would be the selection of a stochastic event rather than the memory of a specific occurrence. As a result, pure epigenetic variation can only play a role in memory when driven by specific environmental circumstances at specific loci or genomic regions, allowing plants to acquire unique expressional states that are beneficial to their fitness as well as the fitness of their progeny. Interestingly, several studies have found that various stressors target epigenetic variation in different chromosomal locations. This discovery implies that some areas of the genome may have been chosen during evolution to retain a type of epigenetic flexibility, maybe as part of a learning process that is beneficial for dynamic adaptation to environmental fluctuation $[2,3,5,7]$.

A number of recent studies have investigated the capacity of genetically identical plant populations to acquire unique environmental-induced epigenetic states, but they found no indication that these modifications may be permanently transmitted. Furthermore, shown that at least two rounds of repeated stress exposure are necessary to allow transgenerational inheritance of induced epigenetic modifications, and that in the absence of a fresh stimulus, these epigenetic abnormalities are reset in following generations. These findings imply that the significance of pure epigenetic variation in plant memory in terms of transmitting learned experience is minor $[2,5,7,23]$.

\section{New definition}

A more accurate definition might be: "epigenetics is the study of the inheritance of information stored within chromatin (hence not linked to the sequence of the DNA double helix) and that persists after the stimulus inducing this information has been removed, independent of the nature of mechanisms mediating its origin (hence the exogenous or endogenous stimulus" $[2,5,20]$.

\section{Discussion and Conclusion}

Plants, like other creatures, can learn and retain what they have learnt. In this process, epigenetic variables may allow knowledge to be passed from generation to generation. Communication allows for the development of learning and memory. As a result, communication creates everlasting liaisons and symbiotic connections with other organisms to comprehend and respond [12,15,21,28,29].

A simple model based on a Hopfield-like network is used by researchers to explain plant intelligence. A group of experts studied the activity of a plant cell as well as its corrective behavior as a network. They built a mathematical associative memory based on the Hopfield model, in which ferromagnetic retrieval and antiferromagnetic terms coexisted, to evaluate the feasibility of the plant network as a memory device. They observed that a single parameter controls the intensity of pattern retrieval disturbance. According to the researchers, the antiferromagnetic structure prevents the system from recalling a pattern. If researchers adjust all weight connections to positive values, this discovery suggests that the plant's memory ability is relatively weak. Researchers, on the other hand, can increase memory capacity by making all the network's connections negative $[4,11,16,19,21,26,31]$.

Outstanding Concerns What is the difference between electrical signaling (seconds) and chemical signaling (hours to days) in terms of altering growth and development in response to disturbance? What function do mutualistic and symbiotic bacteria on roots and leaves play in controlling plant responses to unexpected disturbances? It is known they do so in the long run, but do they also influence plant early decision making, and if so, how? Is plant 'memory' only reliant on epigenetic processes, or does it rely on long-term changes in the chemical signaling network? How does 'memory' come about? [1,2,15,20].

Extensive heritable epigenetic variation in development and morphology contributes significantly to plant growth, morphology, and plasticity variation. Plants not only have memory as a trait of intelligence towards the past, but they can also anticipate everchanging conditions by integrating multifactorial environmental signals, allowing them to maintain adequate behavior in response to varying resource availability and the presence or absence of competitors and attackers $[2,3,5,7,15,16]$. 

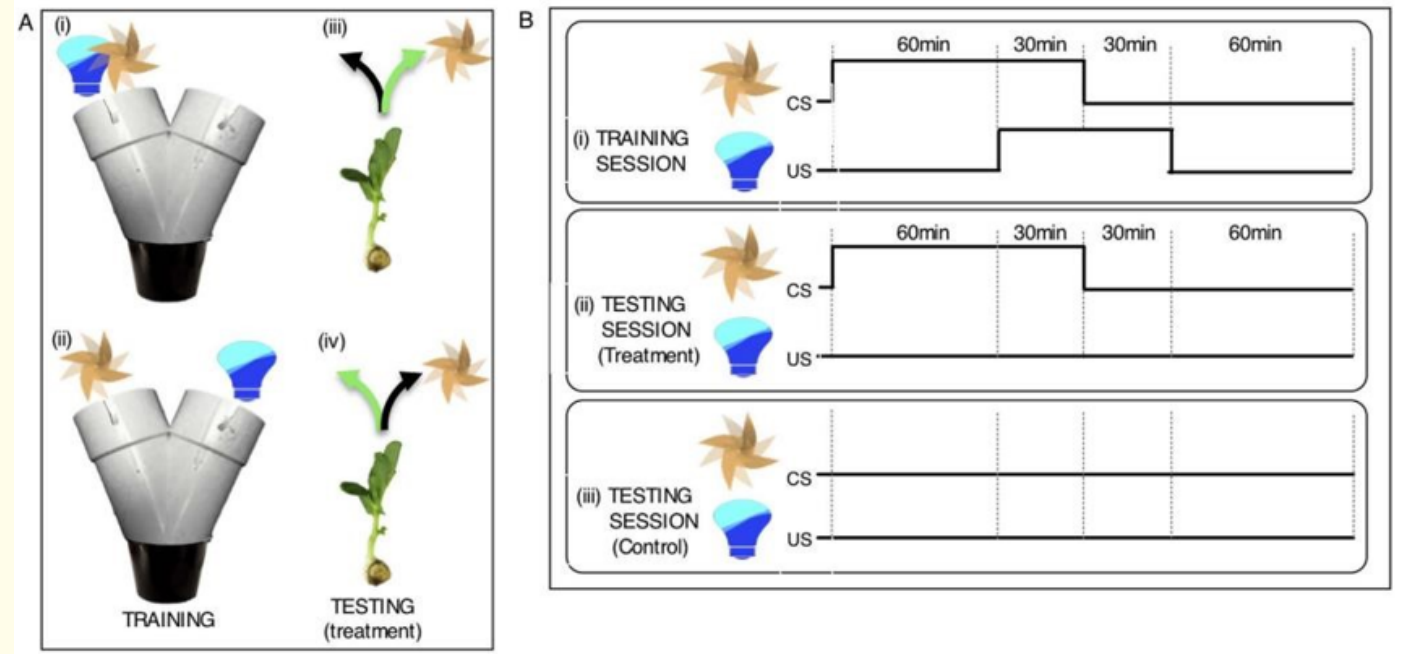

Figure 1: Protocol for training and measuring associative learning in pea seedlings. (A) Seedlings were exposed to the fan [F] and light [L] during training on either the same arm I or the opposite arm (ii) of the Y-maze. The conditioned stimulus (CS) was the fan, while the unconditioned stimulus (US) was light (US). During testing with just the fan, two types of responses emerged. Correct response: Seedlings growing toward the arm of the maze where the fan indicated the light would appear [green arrow; iii (according to scenario I and iv (corresponding to scenario ii)]; Incorrect response: Seedlings growing into the arm of the maze where light was not "predicted" to occur by the fan (black arrow; iii and iv). (B) Before testing, seedlings were trained for three days in a row. Each training day included three 2-hour sessions spaced by 1-hour intervals. The 90-minute CS was 60 minutes ahead of the 60-minute US, resulting in a 30-minute overlap. I Seedlings were exposed to the fan alone for three 90 -minute periods during the one-day testing session (ii). The seedlings in the control group were not disturbed (no fan, no light; iii) [15].
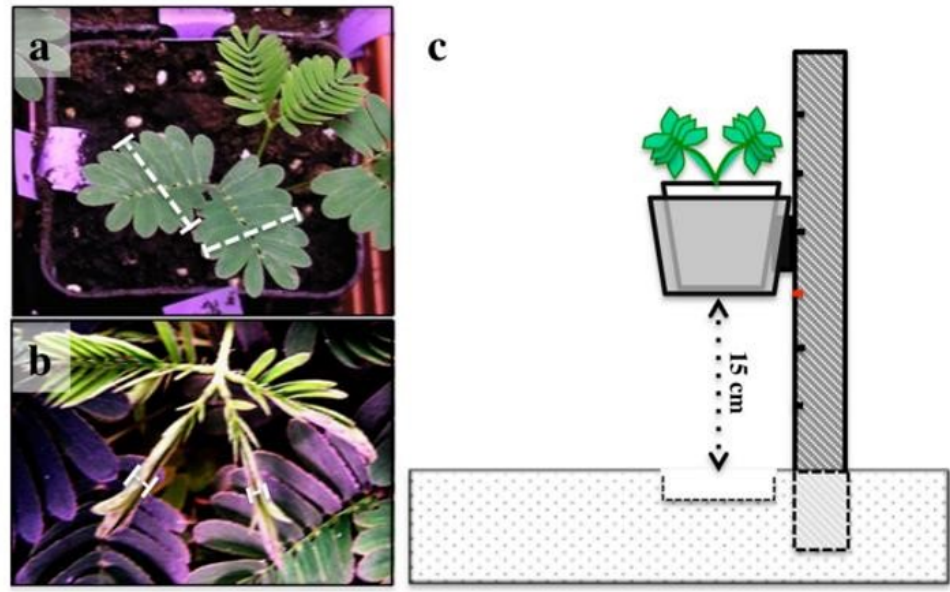

Figure 2: Mimosa plant habituation training using a controlled drop mechanism. Because of its ability to swiftly fold its leaves in reaction to physical perturbation, the sensitive plant Mimosa pudica was chosen as the appropriate model for this investigation. When disturbed, the sub leaflets fold up along the stem of each leaflet, and the leaflet and leaf stem droop downwards in a couple of seconds. The recovery duration varies, and leaves might take anywhere from a few seconds to many minutes to fully re-open. The maximum leaf breadth was measured tip-to-tip before and after training (as indicated by the white dotted line; a and b). A plastic jar was placed with varied hangers onto a designated steel rail, which was then fastened to a foam foundation. Individual potted plants were manually hoisted to the 15 -cm height mark in the host vessel and then allowed to fall by sliding down the rail. The tiny dip in the foam foundation at the vessel's landing site kept it from bouncing on contact. The setup guaranteed that all plants received a consistent amount of perturbation, which was sufficient to cause the closure of all leaves (c) [26]. 


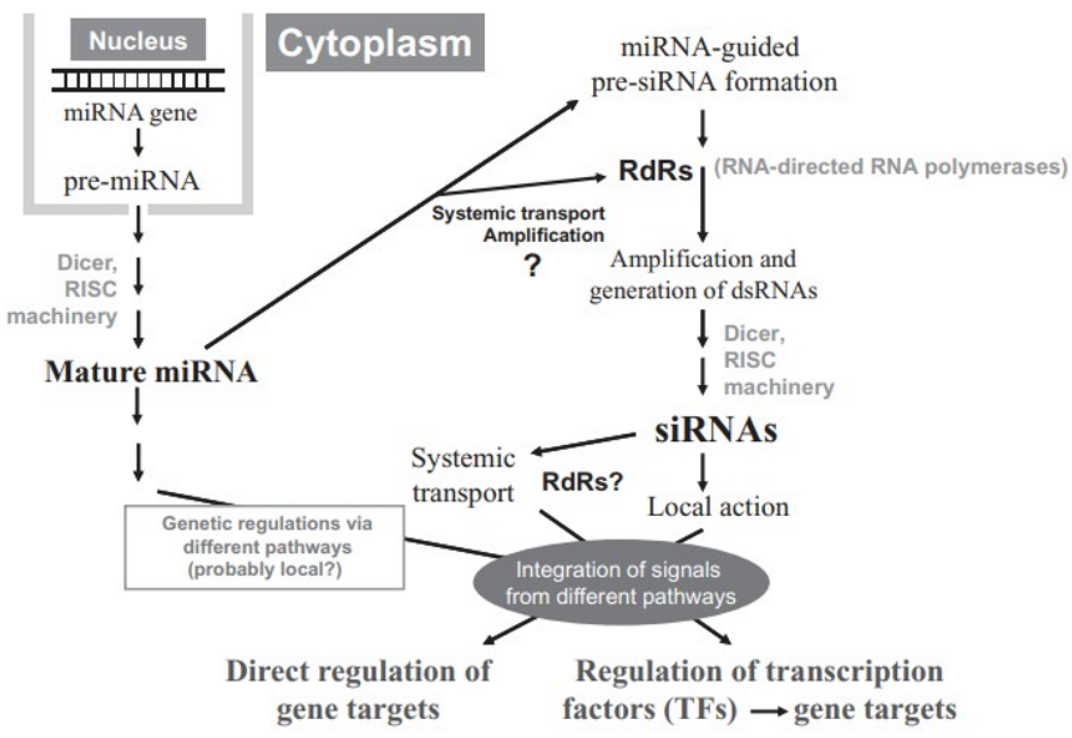

Figure 3: The biosynthesis of short RNAs is depicted in a simplified manner. Small RNAs are classified into two types based on their origins in the nucleus (miRNAs) and the cytoplasm (sRNAs) (siRNAs). To cleave and form mature 20-24 nucleotide smRNAs, both classes rely on Dicer protein and RNA-induced silencing complex (RISC) machinery [25].

\section{Bibliography}

1. Mancuso S and Volkmann D. "Communication in Plants" (2006).

2. Bruce TJA., et al. "Stressful "memories" of plants: Evidence and possible mechanisms". Plant Science 173.6 (2007): 603-608.

3. Baluska., et al. "Signaling and Communication in Plants Memory and Learning in Plants" (2018).

4. Sung S and Amasino RM. "Vernalization and epigenetics: How plants remember winter". Current Opinion in Plant Biology 7.1 (2004): 4-10.

5. van Loon LC. "The Intelligent Behavior of Plants". Trends in Plant Science 21.4 (2016): 286-294.

6. Thellier M., et al. "Long-distance transport, storage and recall of morphogenetic information in plants. The existence of a sort of primitive plant "memory". Comptes rendus de l'Académie des Sciences Ser III 323.1 (2000): 81-91.

7. Van Bel AJE., et al. "Spread the news: Systemic dissemination and local impact of $\mathrm{Ca} 2+$ signals along the phloem pathway". The Journal of Experimental Botany 65.7 (2014): 1761-1787.
8. Rodrigo-Moreno A., et al. "Root phonotropism: Early signalling events following sound perception in Arabidopsis roots". Plant Science 264 (2017): 9-15.

9. Baluška F., et al. "Plant synapses: Actin-based domains for cellto-cell communication". Trends in Plant Science 10.3 (2005): 106-111.

10. White PJ and Broadley MR. "Calcium in plants". Annals of Botany 92.4 (2003): 487-511.

11. Baluška F., et al. "The 'root-brain' hypothesis of Charles and Francis Darwin”. Plant Signaling and Behavior 4.12 (2009): 1121-1127.

12. Gagliano M., et al. "Learning by Association in Plants". Scientific Reports 6 (2016): 1-9.

13. Trewavas A. "What is plant behaviour?" Plant, Cell and Environment 32.6 (2009): 606-616.

14. Gagliano M., et al. "The Language of Plants". Science, Philosophy, Literature (2017).

15. Trewavas A. "Aspects of plant intelligence". Annals of Botany 92.1 (2003): 1-20. 
16. Alpi A., et al. "Plant neurobiology: no brain, no gain?" Trends in Plant Science 12.4 (2007): 135-136.

17. Mallatt J., et al. "Debunking a myth: plant consciousness". Protoplasma 258.3 (2021): 459-476.

18. Trewavas A. "The foundations of plant intelligence". Interface Focus 7.3 (2017): 1-2.

19. Karban R. "Plant behaviour and communication". Ecology Letters 11.7 (2008): 727-739.

20. Thellier M and Lüttge U. "Plant memory: A tentative model". New Phytologist 15.1 (2013): 1-12.

21. Demidchik V., et al. "Calcium transport across plant membranes: mechanisms and functions". New Phytologist 220.1 (2018): 49-69.

22. GÁlis I., et al. "Molecular mechanisms underlying plant memory in JA-mediated defence responses". Plant, Cell and Environment 32.6 (2009): 617-627.

23. Gagliano M., et al. "Experience teaches plants to learn faster and forget slower in environments where it matters". Oecologia 175.1 (2014): 63-72.

24. Gagliano M., et al. "Comment on 'lack of evidence for associative learning in pea plants”. Elife 9 (2020): 1-2.

25. Trewavas T. "Plant intelligence: An overview". Bioscience 66.7 (2016): 542-551.

26. Biegler R. "Insufficient evidence for habituation in Mimosa pudica. Response to Gagliano et al. (2014)". Oecologia 186.1 (2018): 33-35.

27. Trewavas A. "Plant intelligence". Naturwissenschaften 92.9 (2005): 401-413.

28. Inoue J ichi. "A simple Hopfield-like cellular network model of plant intelligence". Progress in Brain Research 168.1998 (2007): 169-174.

29. Demongeot J., et al. "A mathematical model for storage and recall functions in plants". Comptes rendus de l'Académie des Sciences Ser III 323.1 (2007): 93-97.
30. Mchale CM., et al. "Current understanding of the mechanism of benzeneinduced leukemia in humans: Implications for risk assessment". Carcinogenesis 33.2 (2012): 240-252.

\section{Volume 4 Issue 12 December 2021} (C) All rights are reserved by Mehmet Eraslan. 